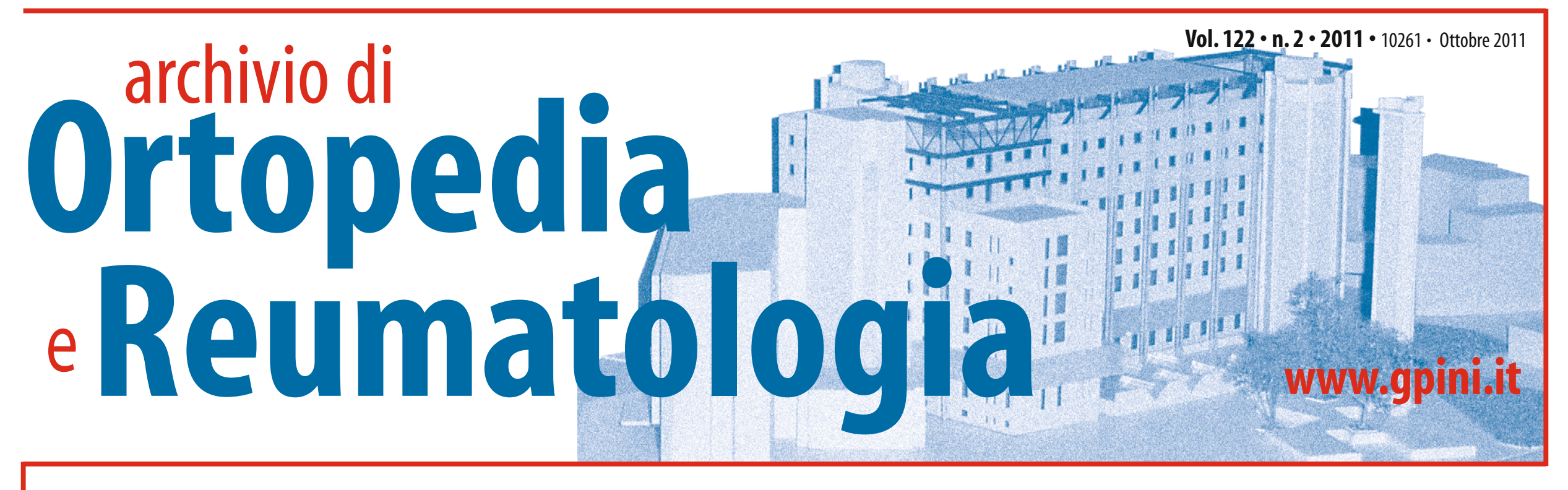

\title{
Le fratture toraco-lombari
}

\section{Editoriale}

a cura di B. Misaggi

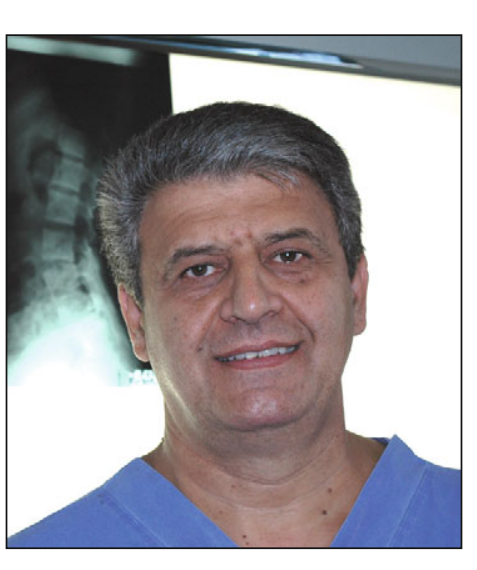

B. Misaggi

e fratture del rachide to-raco-lombare sono spesso la conseguenza di traumatismi a elevata energia e possono sovente essere riscontrate nell'ambito di un paziente politraumatizzato. Approssimativamente il 90\% di tutte le fratture toraciche e lombari si verifica alla giunzione toraco-lombare, che rappresenta dunque il tratto di colonna vertebrale che deve sistematicamente essere indagato clinicamente e radiograficamente in Pronto soccorso in tutti i pazienti che hanno subito un traumatismo vertebrale.

Nonostante il trattamento delle fratture vertebrali sia ancora oggi argomento ampiamente dibattuto in letteratura sia per quanto riguarda l'indicazione alla chirurgia sia sul tipo di approccio chirurgico da adottare, tutti sono concordi nel ritenere indispensabile un adeguato inquadramento classificativo della lesione al fine di poter intraprendere un corretto iter diagnostico e terapeutico. In quest'ottica l'impiego sistematico di una classificazione che sia in grado di contemplare tutte le possibili lesioni fratturative vertebrali e che sia in grado di dare un indirizzo prognostico e terapeutico è indispensabile nell'inquadramento di un paziente affetto da frattura vertebrale. La classificazione, universalmente accettata, che meglio risponde a tali esigenze è quella introdotta da Magerl nel 1994 e che distingue le diverse lesioni vertebrali sulla base del meccanismo traumatico suddividendole in gruppi e sottogruppi a crescente gravità lesionale.

Un corretto inquadramento clinico e radiografico e l'utilizzo della classificazione di Magerl permettono di ben distinguere le lesioni stabili da quelle instabili che necessariamente richiedono un intervento chirurgico.

Il trattamento conservativo delle fratture vertebrali ha ancora oggi un ruolo nella gestione del paziente affetto da frattura vertebrale isolata e non complicata, ma le crescenti richieste funzionali e i rischi dell'allettamento hanno fatto sì che un numero sempre maggiore di pazienti rifiuti il trattamen- to conservativo classico basato sull'utilizzo del corsetto gessato in iperestensione rachidea e sullo scarico assoluto a letto.

Inoltre le nuove tecniche chirurgiche mini-invasive di vertebro- e cifoplastica, in aggiunta alla possibilità di effettuare interventi chirurgici di stabilizzazione vertebrale percutanea, hanno ampliato notevolmente le indicazioni al trattamento chirurgico, riducendo drasticamente i rischi connessi all'allettamento forzato e le deformità vertebrali posttraumatiche.

Il trattamento chirurgico delle fratture vertebrali ha infatti come obiettivo primario il ripristino della stabilità segmentaria consentendo una precoce mobilizzazione del paziente e prevenendo i rischi dell'allettamento e degli esiti invalidanti in cifosi del rachide dorso-lombare. Tali obiettivi di trattamento possono essere facilmente raggiunti nella stragrande maggioranza dei casi con una stabilizzazione vertebrale eseguita per via posteriore, di rapida e sicura esecuzione e gravata da un basso rischio di complicanze. La fissazione vertebrale per via posteriore può essere effettuata facilmente anche con tempistiche di urgenza quando può risultare difficoltoso organizzare un intervento chirurgico per via anteriore toracotomica, lombotomica o attraverso una toracofrenolombotomia.

Laccesso anteriore va riservato a casi attentamente selezionati in cui è necessaria un'ampia decompressione canalare oppure una completa ricostruzione somati$\mathrm{ca}$, come intervento isolato oppure a distanza di tempo variabile da una precedente fissazione posteriore, nel caso in cui è realmente necessario.

Vorrei infine ricordare come ogni metodica chirurgica di stabilizzazione di una frattura vertebrale, così come di qualsiasi altra frattura scheletrica, deve avere come obiettivo primario la guarigione biologica della frattura stessa o, nel caso della colonna vertebrale, l'ottenimento di una solida spondilodesi, oltre a un perfetto "balance" sagittale. Tutto ciò può essere solo ottenuto con una adeguata riduzione e stabilizzazione della frattura abbinata all'impiego di materiali biologici (innesto osseo autologo o omologo) o di sostituti dell'osso, e non con un irrazionale utilizzo del cemento acrilico, il cui impiego deve essere limitato alle fratture da impoverimento osseo dell'anziano e non alle fratture traumatiche del giovane.

B. Misaggi UOC di Chirurgia Vertebrale e Scoliosi, Istituto Ortopedico G. Pini, Milano 